\title{
Aut-invariant quasimorphisms on free products
}

\author{
Bastien Karlhofer ${ }^{1}$
}

Received: 25 March 2021 / Accepted: 5 July 2021

(c) The Author(s) 2021

\begin{abstract}
Let $G=A * B$ be a free product of freely indecomposable groups. We explicitly construct quasimorphisms on $G$ which are invariant with respect to all automorphisms of $G$. We also prove that the space of such quasimorphisms is infinite-dimensional whenever $G$ is not the infinite dihedral group. As an application we prove that an invariant analogue of stable commutator length recently introduced by Kawasaki and Kimura is non-trivial for these groups.

\section{Résumé}

Soit $G=A * B$ un produit libre de groupes librement indécomposables. Nous construisons explicitement des quasimorphismes sur $G$ qui sont invariants par rapport à tous les automorphismes de $G$. Nous prouvons également que l'espace de tels quasimorphismes est de dimension infinie lorsque $G$ n'est pas le groupe dièdre infini. En tant qu'application, nous prouvons qu'un analogue invariant de longueur de commutateur stable récemment introduit par Kawasaki et Kimura est non trivial pour ces groupes.
\end{abstract}

Keywords Aut-invariant · Quasimorphism · Free product · Stable commutator length

Mathematics Subject Classification 20F65 · 20E06

\section{Introduction}

The study of quasimorphisms on a given group $G$ is an important branch of geometric group theory [6] with quasimorphisms sharing deep relationships with the underlying structure of the group $G$. For free groups $F_{n}$ the so called counting quasimorphisms originating from the work of Brooks in [4] yield a wide variety of examples. His ideas have been developed further by Calegari and Fujiwara who constructed unbounded quasimorphisms on non-elementary hyperbolic groups [5]. For diffeomorphism groups of surfaces many important constructions are given in [9]. There are also numerous applications in symplectic geometry originating from work of Entov and Polterovich [7]. Another fundamental paper on the geometry of quasimorphisms and central extensions is [1].

$凶$ Bastien Karlhofer

r01bdk17@abdn.ac.uk

1 University of Aberdeen, Aberdeen, UK 
In this paper we construct unbounded Aut-invariant quasimorphisms on free products of groups. To achieve this we associate tuples of natural numbers we call codes to each element in a free product $G=A * B$. Inspired by Brooks counting quasimorphisms on free groups we then count these codes rather than actual elements of the free product and verify that this indeed yields quasimorphisms on $G$. We call them code quasimorphisms. We make use of an explicit description of the automorphism group of a free product found in [8] to see in Proposition 4.11 that our code quasimorphisms are unbounded and invariant with respect to all automorphisms of $G$ if $A$ and $B$ are not infinite cyclic.

If one of the factors of $G=A * B$ is infinite cyclic, our code quasimorphism are not necessarily invariant under a specific class of automorphisms of $G$ which is called the class of transvections. So, we slightly adjust the way we count codes for infinite cyclic factors and call the resulting maps weighted code quasimorphisms. We show in Proposition 5.7 that these are unbounded and invariant with respect to all automorphisms of $G$.

These two propositions together with an independent result for the free group on two generators from [3, Theorem 2] comprise the following result in Sect. 6 which is the main result of the paper.

Theorem 1 Let $G=A * B$ be the free product of two non-trivial freely indecomposable groups $A$ and $B$. Assume $G$ is not the infinite dihedral group. Then $G$ admits infinitely many linearly independent homogeneous Aut-invariant quasimorphisms, all of which vanish on single letters.

The infinite dihedral group does not admit any unbounded quasimorphism since all its elements are conjugate to their inverses. As a corollary of our construction we immediately deduce the existence of stably unbounded Aut-invariant norms on free products of two factors.

Corollary 1.1 Let $G=A * B$ be the free product of two non-trivial freely indecomposable groups and assume $G$ is not the infinite dihedral group. Then there exists a stably unbounded Aut-invariant norm on $G$.

We denote the Aut-invariant stable commutator length, which was recently introduced by Kawasaki and Kimura in [12], by $\mathrm{scl}_{\text {Aut }}$. As an application of our construction we prove the following result in Sect. 7.

Theorem 2 Let $G=A * B$ be a free product of freely indecomposable groups and assume that $G$ is not the infinite dihedral group. Then there always exist elements $g \in G$ with positive Aut-invariant stable commutator length $\mathrm{scl}_{\mathrm{Aut}}(g)>0$.

\section{Preliminaries}

Definition 2.1 For any group $G$ we denote by $\operatorname{Aut}(G)$ the group of all automorphisms of $G$. Moreover, we denote the normal subgroup of inner automorphisms by $\operatorname{Inn}(G)$ and define the group of outer automorphisms of $G$ to be the quotient $\operatorname{Out}(G)=\operatorname{Aut}(G) / \operatorname{Inn}(G)$.

Definition 2.2 Let $G$ be a group. A map $\psi: G \rightarrow \mathbb{R}$ is called a quasimorphism if there exists a constant $D \geq 0$ such that

$$
|\psi(g)+\psi(h)-\psi(g h)| \leq D \quad \text { for all } g, h \in G .
$$

The smallest number $D(\psi)$ with the above property is called the defect of $\psi$. We call a quasimorphism homogeneous if it satisfies $\psi\left(g^{n}\right)=n \psi(g)$ for all $g \in G$ and all $n \in \mathbb{Z}$. If $\psi(\varphi(g))=\psi(g)$ for all $g \in G, \varphi \in \operatorname{Aut}(G)$, then $\psi$ is called Aut-invariant. 
Definition 2.3 Let $\psi: G \rightarrow \mathbb{R}$ be a quasimorphism. Then the homogenisation $\bar{\psi}: G \rightarrow \mathbb{R}$ of $\psi$ is defined by $\bar{\psi}(g)=\lim _{n \in \mathbb{N}} \frac{\psi\left(g^{n}\right)}{n}$ for all $g \in G$.

Lemma 2.4 ( $[6, \mathrm{p} .18])$ The homogenisation $\bar{\psi}$ of a quasimorphism $\psi: G \rightarrow \mathbb{R}$ is a homogeneous quasimorphism. Moreover, it satisfies $|\bar{\psi}(g)-\psi(g)| \leq D(\psi)$ for any $g \in G$.

Definition 2.5 A function $v: G \rightarrow \mathbb{R}$ satisfying for all $g, h \in G$ :

- $v(g) \geq 0$,

- $v(g)=0$ if and only if $g=1$,

- $v(g h) \leq v(g)+v(h)$,

is called a norm on $G$. If in addition for all $g \in G$ and $\varphi \in \operatorname{Aut}(G)$ it satisfies

- $v(\varphi(g))=v(g)$,

then it is called Aut-invariant. The supremum $v(G)=\sup \{v(g) \mid g \in G\}$ is called the diameter of the norm $v$. If $v(G)=\infty$, then $v$ is called unbounded. If there exists $g \in G$ such that $\lim _{n \rightarrow \infty} \frac{v\left(g^{n}\right)}{n}>0$, then $v$ is called stably unbounded.

Example 2.6 Let $G$ be a group together with a generating set $S$. The word norm generated by $S$ is the norm on $G$ defined by

$$
v_{S}(g)=\min \left\{n \mid g=s_{1} \ldots s_{n} \text { where } n \in \mathbb{N} \text { and } s_{i} \in S \text { for all } i\right\} .
$$

If we assume additionally that the set $S$ is invariant under $\operatorname{Aut}(G)$ then $v_{S}$ is Aut-invariant.

Lemma 2.7 Let $\psi: G \rightarrow R$ be an Aut-invariant quasimorphism with unbounded image, but bounded on a generating set $S$ of $G$. Then there exists a stably unbounded Aut-invariant norm on $G$.

Proof By Lemma 2.4 we can assume that $\psi$ is homogeneous. The word norm $\|\cdot\|_{\bar{S}}$ on $G$ associated to the generating set $\bar{S}=\{\varphi(s) \mid s \in S, \varphi \in \operatorname{Aut}(G)\}$ is clearly Aut-invariant. Let $K$ be a positive bound for the absolute value of $\psi$ on $S$. Write $g \in G$ as a product $g=\varphi_{1}\left(s_{1}\right) \ldots \varphi_{n}\left(s_{n}\right)$ for some $n \in \mathbb{N}$ where $s_{i} \in S$ and $\varphi_{i} \in \operatorname{Aut}(G)$ for all $i$. The calculation

$$
\begin{aligned}
& |\psi(g)|=\left|\psi\left(\varphi_{1}\left(s_{1}\right) \ldots \varphi_{n}\left(s_{n}\right)\right)\right| \leq\left|\psi\left(\varphi_{1}\left(s_{1}\right)\right)\right|+\cdots+\left|\psi\left(\varphi_{n}\left(s_{n}\right)\right)\right|+(n-1) D(\psi) \\
& \quad \leq n(K+D(\psi))
\end{aligned}
$$

shows that $\|g\|_{\bar{S}} \geq \frac{|\psi(g)|}{K+D(\psi)}$ for all $g \in G$. It follows that $\left\|g^{k}\right\|_{\bar{S}} \geq k \cdot \frac{|\psi(g)|}{K+D(\psi)}$ for all $k \in \mathbb{N}$, $g \in G$. Since $\psi$ does not vanish everywhere, $\|\cdot\|_{\bar{S}}$ is a stably unbounded Aut-invariant norm on $G$.

The following examples illustrate that the converse of Lemma 2.7 above is not true and finding unbounded Aut-invariant quasi-morphisms is much more difficult than finding unbounded Aut-invariant norms.

Example 2.8 Let $\Sigma_{\infty}$ be the infinite symmetric group of finitely supported bijections of the natural numbers. The cardinality of the support defines an Aut-invariant norm of infinite diameter on $\Sigma_{\infty}$. However, any element $g \in \Sigma_{\infty}$ has finite order. Therefore, no Aut-invariant norm on $\Sigma_{\infty}$ is stably unbounded and any homogeneous quasi-morphism vanishes on all of $\Sigma_{\infty}$. Consequently, by Lemma 2.4 any quasimorphism on $\Sigma_{\infty}$ is bounded. 
Example 2.9 Let $G=\mathbb{Z}^{k}$ for $k \geq 1$. Since every $g \in G$ lies in the same $\operatorname{Aut}(G)$-orbit as $g^{-1}$, it follows that any homogeneous Aut-invariant quasimorphism vanishes on all of $G$. So any Aut-invariant quasimorphism is bounded on $G$. For $k=1$ the standard absolute value defines a stably unbounded Aut-invariant norm on $G$, whereas for $k \geq 2$ any Aut-invariant norm on $G$ has finite diameter.

Example 2.10 Let $G$ be the fundamental group of the Klein bottle $G=\mathbb{Z} *_{2 \mathbb{Z}} \mathbb{Z}$. Let $a$ and $b$ be generators of the two infinite cyclic factors of $G$ in its above presentation. Consider the Aut-invariant word norm $v_{S}$ generated by $S=\left\{\varphi\left(a^{ \pm 1}\right), \varphi\left(b^{ \pm 1}\right) \mid \varphi \in \operatorname{Aut}(G)\right\}$. To see that this norm is unbounded on $G$ we first note that commutator subgroup of $G$ is a characteristic subgroup and $G /[G, G]=\mathbb{Z} / 2 \times \mathbb{Z}$, where $\mathbb{Z} / 2$ is a characteristic subgroup again. Consequently, the projection map $p: G \rightarrow \mathbb{Z}$ sending $p(a)=p(b)=1$ maps the set $S$ to the Aut-invariant set $\{ \pm 1\}$ in $\mathbb{Z}$, which generates a stably unbounded Aut-invariant norm on $\mathbb{Z}$. Therefore, the word norm $v_{S}$ generated by $S$ on $G$ is stably unbounded as well.

However, there is no unbounded Aut-invariant quasimorphism on $G$. If $\psi$ was such a quasimorphism, it could be chosen to be homogeneous by Lemma 2.4. Let $\varphi$ be the automorphism inverting the generators $a$ and $b$. Then $\varphi$ inverts the center $Z(G)=2 \mathbb{Z}$ as well. Hence, $\psi$ vanishes on $Z(G)$. Similarly, every element of $S=\left\{(a b)^{n},(b a)^{n},(a b)^{n} a,(b a)^{n} b \mid n \in \mathbb{N}\right\}$ belongs to the same Aut-orbit that its inverse belongs to. So $\psi$ vanishes on $S$ as well. However, every element $g \in G$ can be written as a product $=z s$ where $z \in Z(G)$ and $s \in S$. Therefore, $\psi$ is bounded on all of $G$.

Definition 2.11 Let $G=*_{i \in I} G_{i}$ be a free product of a family of groups $\left\{G_{i}\right\}_{i \in I}$ for some indexing set $I$. For each $i$ the factor $G_{i}$ is a subgroup of $G$ via the canonical inclusion. An element of $G$ that belongs to one of the factors is called a letter of $G$. Any product of letters is called a word in $G$. The product of any two letters belonging to the same factor in $G$ can be replaced by the letter that represents their product in that factor. Moreover, any identity letters appearing in a word can be omitted without changing the element the word represents in $G$. Recall that any element $g \in G$ has a unique presentation as a word, where no two consecutive letters lie in the same factor and no identity letters appear. Such a word is called reduced.

Lemma 2.12 Let I be a set of cardinality at least two. Let $G_{i}$ be a non-trivial group for all $i$ and $G=*_{i \in I} G_{i}$ be their free product. Let $\theta: G \rightarrow \mathbb{R}$ be a map whose absolute value is bounded on all letters of $G$ by a constant $B \geq 0$. Assume that there exists a constant $D \geq 0$ such that

$$
\left|\theta\left(w_{1} w_{2}\right)-\theta\left(w_{1}\right)-\theta\left(w_{2}\right)\right| \leq D
$$

holds for all reduced words $w_{1}, w_{2}$ for which their product $w_{1} w_{2}$ is a reduced word. Then the map $f: G \rightarrow \mathbb{R}$ defined by $f(w)=\theta(w)-\theta\left(w^{-1}\right)$ defines a quasimorphism of defect at most $12 D+6 B$, which is bounded on all letters by $2 B$.

Proof Any element in $G$ can be represented by a reduced word. So let $w_{1}, w_{2}$ be reduced words. The word given by their product $w_{1} w_{2}$ is reduced if and only if the last letter from $w_{1}$ belongs to a factor different from the one that the first letter of $w_{2}$ belongs to. Indeed, otherwise those two letters could be multiplied in their common factor and replaced by their product to shorten the number of letters appearing in the expression.

In order to bring $w_{1} \cdot w_{2}$ to its reduced form we first perform all cancellations which form a word we call $c$. After all cancellations have taken place the final potential reduction is to possibly replace a non-trivial product of two letters $b$ and $d$ belonging to the same factor by a non-trivial letter $x$ representing their product in that factor. Therefore, we have two cases. 
- The reduced presentations of $w_{1}$ and $w_{2}$ are given by $w_{1}=a c, w_{2}=c^{-1} e$ and $a e$ is the reduced presentation for $w_{1} \cdot w_{2}$.

- The reduced presentations of $w_{1}$ and $w_{2}$ are given by $w_{1}=a b c, w_{2}=c^{-1} d e$, where $b$ and $d$ are letters belonging to the same factor. The reduced presentation of $w_{1} \cdot w_{2}$ is given by axe, where $x=b d$ is the letter representing the non-trivial product of $b$ and $d$.

We calculate for the second case that

$$
\begin{aligned}
& \left|f\left(w_{1} w_{2}\right)-f\left(w_{1}\right)-f\left(w_{2}\right)\right|=\mid f(\text { axe })-f(a b c)-f\left(c^{-1} d e\right) \mid \\
& =\mid \theta(\text { axe })-\theta\left(e^{-1} x^{-1} a^{-1}\right)-\theta(a b c)+\theta\left(c^{-1} b^{-1} a^{-1}\right)-\theta\left(c^{-1} d e\right) \\
& \quad+\theta\left(e^{-1} d^{-1} c\right) \mid \\
& \leq \mid \theta(a)+\theta(x)+\theta(e)-\theta\left(e^{-1}\right)-\theta\left(x^{-1}\right)-\theta\left(a^{-1}\right)-\theta(a)-\theta(b)-\theta(c)+\theta\left(c^{-1}\right) \\
& +\theta\left(b^{-1}\right)+\theta\left(a^{-1}\right)-\theta\left(c^{-1}\right)-\theta(d)-\theta(e)+\theta\left(e^{-1}\right)+\theta\left(d^{-1}\right) \\
& \quad+\theta(c) \mid+12 D \\
& =\left|\theta(x)-\theta\left(x^{-1}\right)-\theta(b)+\theta\left(b^{-1}\right)-\theta(d)+\theta\left(d^{-1}\right)\right|+12 D \\
& \leq 6 B+12 D .
\end{aligned}
$$

The first case follows analogously. Since $w_{1}, w_{2}$ were arbitrary reduced words and every element of $G$ can be written in its reduced form, $f$ is a quasimorphism of defect at most $6 B+12 D$. Since $\theta$ is bounded on all letters by $B$, so is $f$ by $2 B$.

\section{Aut-invariant quasimorphisms}

Definition 3.1 We call a group $G$ freely indecomposable if $G$ is non-trivial and not isomorphic to any free product of the form $G_{1} * G_{2}$ where $G_{1}, G_{2}$ are non-trivial groups.

Any free product of non-trivial groups has trivial center and contains elements of infinite order. So every abelian group and every finite group is freely indecomposable.

Lemma 3.2 Let $\psi: G \rightarrow \mathbb{R}$ be a quasimorphism. Let $\left\{\varphi_{i}\right\}_{i \in I}$ be a set of representatives for the elements of $\operatorname{Out}(G)$. If $\psi$ is invariant under $\varphi_{i}$ for all $i$, then its homogenisation $\bar{\psi}: G \rightarrow \mathbb{R}$ is invariant under all automorphisms of $G$.

Proof The homogenisation $\bar{\psi}$ is constant on conjugacy classes [6, p.19]. By definition $\bar{\psi}$ is invariant under the collection $\left\{\varphi_{i}\right\}_{i \in I}$, since $\psi$ is. The result follows since any element $\varphi \in \operatorname{Aut}(G)$ can be written as the composition of some $\varphi_{j}$ with a conjugation.

Consider the free product $G=G_{1} * G_{2}$ where $G_{i}$ is freely indecomposable for $i=1,2$. Following the exposition in [8, p.116] based on results in [10] and [11] the automorphism group $\operatorname{Aut}\left(G_{1} * G_{2}\right)$ is generated by the following types of automorphisms (1-3) if neither $G_{1}$ nor $G_{2}$ is infinite cyclic:

1. Elements from $\operatorname{Aut}\left(G_{1}\right)$ and $\operatorname{Aut}\left(G_{2}\right)$ give rise to automorphisms of $G_{1} * G_{2}$. These are called factor automorphisms.

2. Let $g \in G_{i}$ for some $i \in\{1,2\}$. Define the map $p_{g}: G \rightarrow G$ to be conjugation by $g$ on the letters of $G_{j}$ for $j \neq i$ and to be the identity on all letters from the group $G_{i}$. This definition gives rise to an automorphism of $G$ which is called a partial conjugation.

3. If $G_{1} \cong G_{2}$ are isomorphic, interchanging the two factors is an automorphism of $G$. Such an automorphism is called a swap automorphism. 
If $G_{1} \cong \mathbb{Z}$ is infinite cyclic and the freely indecomposable group $G_{2}$ is not, then $\operatorname{Aut}\left(G_{1} * G_{2}\right)$ is generated by the above automorphisms together with the following additional type of automorphisms:

4. Let $s$ be a generator of $G_{1}$ and let $a \in G_{2}$ be any element. Then a transvection is the unique automorphism of $G_{1} * G_{2}$ defined to be the identity on all letters from $G_{2}$ and maps $s \rightarrow$ as or $s \rightarrow s a$.

Following the above description of the group of automorphisms of a free product of two factors we obtain:

Lemma 3.3 Let $G_{1}, G_{2}$ be freely indecomposable groups such that $G_{2}$ is not infinite cyclic. Then the outer automorphism group of their free product $\operatorname{Out}\left(G_{1} * G_{2}\right)$ is generated by the images of $\operatorname{Aut}\left(G_{1}\right)$, Aut $\left(G_{2}\right)$ in $\operatorname{Out}\left(G_{1} * G_{2}\right)$ together with a swap automorphism if $G_{1} \cong G_{2}$ and the transvections if $G_{1} \cong \mathbb{Z}$.

Proof By the universal property of the free product of two groups any automorphism is uniquely determined by its image on single letters. Let $h \in G_{1}$ and denote conjugation by $h^{-1}$ on all of $G$ by $c_{h}$. Then

$$
\left(c_{h} \circ p_{h}\right)(g)= \begin{cases}h^{-1} g h & \text { if } g \in G_{1}, \\ g & \text { if } g \in G_{2} .\end{cases}
$$

Thus, $p_{h}$ and the factor automorphism given by conjugation by $h^{-1}$ on $G_{1}$ represent the same element in $\operatorname{Out}(G)$. Similarly, in $\operatorname{Out}(G)$ partial conjugations on $G_{1}$ by elements from $G_{2}$ represent the same elements that factor automorphisms from $G_{2}$ do. Finally, any two choices of swap automorphism differ by a product of factor automorphisms.

Lemma 3.4 Let $G$ be a group and $H \leq G$ be a characteristic subgroup with quotient projection $p: G \rightarrow G / H$. Then for any unbounded Aut-invariant quasimorphism $\psi: G / H \rightarrow \mathbb{R}$ the composition $\psi \circ p: G \rightarrow \mathbb{R}$ is an unbounded Aut-invariant quasimorphism on $G$. Moreover, linearly independent quasimorphisms on $\mathrm{G} / \mathrm{H}$ give rise to linearly independent quasimorphisms on $G$.

Proof Clearly, $\psi \circ p$ is a quasimorphism. The Aut-invariance of $\psi \circ p$ on $G$ follows from the Aut-invariance of $\psi$ on $G / H$ together with the fact that $H$ is characteristic. Finally, the statement about linear independence follows from the surjectivity of the projection to the quotient.

\section{Code quasimorphisms}

Recall that a tuple always refers to a finite sequence and so all tuples are naturally ordered.

Definition 4.1 Let $A$ and $B$ be groups. Write a given element $g \in A * B$ in its reduced form. We assign two tuples of non-zero natural number that we will call codes as follows. Let $\left(a_{1}, \ldots, a_{k}\right)$ be the tuple of letters from $A$ appearing in the reduced form of $g$. We call $\left(a_{1}, \ldots, a_{k}\right)$ the $A$-tuple of $g$. Then we count how often any one letter of $\left(a_{1}, \ldots, a_{k}\right)$ appears consecutively. This yields a tuple of positive numbers A-code $(g)=\left(n_{1}, n_{2}, \ldots, n_{r}\right)$ which we call the $A$-code of $g$. Similarly, we obtain the $B$-tuple, which is the tuple of letters from $B$ appearing in the reduced form of $g$, and the $B$-code of $g$, denoted B-code $(g)$, by counting consecutive appearances of letters in the $B$-tuple. 
Note that $\mathrm{A}-\operatorname{code}(g)$ and $\mathrm{B}-\operatorname{code}(g)$ might have very different length for elements $g \in A * B$ in general.

Example 4.2 Let $G=A * B$ where $A=\mathbb{Z} / 5$ and $B$ is any group. Let $a \in A, b \in B$ be non-trivial elements. Consider $g=a^{2} b a b a b a^{4} b a b a$. The $A$-tuple of $g$ is $\left(a^{2}, a, a, a^{4}, a, a\right)$ and therefore $\mathrm{A}$-code $(g)=(1,2,1,2)$. However, the $B$-tuple of $g$ is $(b, b, b, b, b)$ and so B-code $(g)=(5)$.

Remark 4.3 The code of any element $g \in A * B$ is clearly invariant under all factor automorphisms.

The following lemma is immediate.

Lemma 4.4 The A-code and $\mathrm{B}$-code of $g^{-1}$ are the reversed $A$ - and $\mathrm{B}$-code of $g$ for any $g \in A * B$. That is, let $\mathrm{A}-\operatorname{code}(g)=\left(n_{1}, \ldots, n_{k}\right)$ and $\mathrm{B}-\operatorname{code}(g)=\left(m_{1}, \ldots, m_{\ell}\right)$, then $\mathrm{A}-\operatorname{code}\left(g^{-1}\right)=\left(n_{k}, \ldots, n_{1}\right)$ and $\mathrm{B}-\operatorname{code}\left(g^{-1}\right)=\left(m_{\ell}, \ldots, m_{1}\right)$.

In the spirit of Brooks counting quasimorphisms we will now define code quasimorphisms, which are counting the occurrences of a string of natural numbers in the A-code and B-code associated to an element in the free product $A * B$.

Definition 4.5 (Code quasimorphisms) Let $k \geq 1$ and let $z=\left(n_{1}, \ldots, n_{k}\right)$ be a tuple of nonzero natural numbers $n_{1}, \ldots, n_{k}$ for some $k \in \mathbb{N}$. Let $C \in\{A, B\}$. Define $\theta_{z}^{C}: A * B \rightarrow \mathbb{Z}_{\geq 0}$ to count the maximal number of disjoint appearances of $z$ as a tuple of consecutive numbers in the $\mathrm{C}$-code for all $g \in A * B$. Further, define the code quasimorphism

$$
f_{z}^{C}: A * B \rightarrow \mathbb{Z} \quad \text { by } \quad f_{z}^{C}(g)=\theta_{z}^{C}(g)-\theta_{z}^{C}\left(g^{-1}\right)
$$

for all $g \in A * B$. Note that $\theta_{z}^{C}\left(g^{-1}\right)=\theta_{\bar{z}}^{C}(g)$ due to Lemma 4.4, where $\bar{z}$ denotes the reversed tuple $\left(n_{k}, \ldots, n_{1}\right)$. Consequently, $f_{z}^{C}(g)$ can also be written as $f_{z}^{C}(g)=\theta_{z}^{C}(g)-\theta_{\bar{z}}^{C}(g)$ for all $g \in G$.

Example 4.6 Let $G=\mathbb{Z} / 5 * B$ and $g=a^{2}$ bababa $a^{4} b a b a$ for non-trivial $a \in A, b \in B$ as in Example 4.2. For $z=(1,2)$ we calculate $\theta_{z}^{A}(g)=2$ and $\theta_{z}^{A}\left(g^{-1}\right)=\theta_{\bar{z}}^{A}(g)=1$ and so $f_{z}^{A}(g)=2-1=1$.

Example 4.7 Let $G=\mathbb{Z} / 5 * B$ and $g=a^{4} b a b a b a^{3} b a b a b a^{3}$ for non-trivial $a \in A, b \in B$. In this case $A-\operatorname{code}(g)=(1,2,1,2,1)$. Then $\theta_{z}^{A}(g)=1$ for $z=(1,2,1)$ since we only count disjoint occurrences. Similarly, $\theta_{z}^{A}\left(g^{-1}\right)=\theta_{\bar{z}}^{A}(g)=1$ and so $f_{z}^{A}(g)=0$.

Lemma 4.8 Let $A, B$ be non-trivial groups and let $C \in\{A, B\}$. For a non-empty tuple of nonzero natural numbers $z$ the map $f_{z}^{C}: A * B \rightarrow \mathbb{Z}$ defines a quasimorphism that is bounded on letters and invariant with respect to all factor automorphisms. Moreover, $D\left(f_{z}^{C}\right) \leq 30$.

Proof We want to apply Lemma 2.12 to deduce that $f_{z}^{C}$ is a quasimorphism. Clearly, $\left|\theta_{z}^{C}(x)\right| \leq 1$ for all letters $x \in A * B$ and all $z$. Let $w_{1}, w_{2}$ be reduced words representing elements in $A * B$ such that their product $w_{1} w_{2}$ is reduced. That is, the last letter of $w_{1}$ and the first letter of $w_{2}$ belong to different factors. Without loss of generality we can assume $C=A$. Let A-code $\left(w_{1}\right)=\left(n_{1}, \ldots, n_{k}\right)$ and A-code $\left(w_{2}\right)=\left(m_{1}, \ldots, m_{\ell}\right)$. Let $x$ be the last letter from $A$ in $w_{1}$ and let $y$ be the first letter from $A$ in $w_{2}$. Then

$$
\mathrm{A}-\operatorname{code}\left(w_{1} w_{2}\right)= \begin{cases}\left(n_{1}, \ldots, n_{k}, m_{1}, \ldots, m_{\ell}\right) & \text { if } x \neq y, \\ \left(n_{1}, \ldots n_{k-1}, n_{k}+m_{1}, m_{2}, \ldots, m_{\ell}\right) & \text { if } x=y .\end{cases}
$$


If $x \neq y$, then $\theta_{z}^{C}\left(w_{1} w_{2}\right) \in\left\{\theta_{z}^{C}\left(w_{1}\right)+\theta_{z}^{C}\left(w_{2}\right), \theta_{z}^{C}\left(w_{1}\right)+\theta_{z}^{C}\left(w_{2}\right)+1\right\}$ since at most one of the disjoint occurrences of $z$ can involve numbers that do not lie completely in the A-code of either $w_{1}$ or $w_{2}$.

If $x=y$, then $\theta_{z}^{C}\left(w_{1} w_{2}\right) \geq \theta_{z}^{C}\left(w_{1}\right)+\theta_{z}^{C}\left(w_{2}\right)-2$ since $n_{k}$ and $m_{1}$ can each be contained in at most one occurrences of $z$ in the A-code of $w_{1}$ and $w_{2}$. Moreover, if an occurrence of $z$ in the A-code of $w_{1} w_{2}$ involves $n_{k}+m_{1}$, then all other occurrences are fully contained in either the A-code of $w_{1}$ or $w_{2}$. Thus, $\theta_{z}^{C}\left(w_{1} w_{2}\right) \leq \theta_{z}^{C}\left(w_{1}\right)+\theta_{z}^{C}\left(w_{2}\right)+1$.

In both cases we conclude

$$
\left|\theta_{z}^{C}\left(w_{1} w_{2}\right)-\theta_{z}^{C}\left(w_{1}\right)-\theta_{z}^{C}\left(w_{2}\right)\right| \leq 2,
$$

and it follows from Lemma 2.12 that $f_{z}^{C}$ is a quasimorphism of defect $D\left(f_{z}^{C}\right) \leq 30$.

Moreover, by Remark 4.3 the maps $\theta_{z}^{C}$ are invariant under all factor automorphisms of $A * B$. Consequently, $f_{z}^{C}=\theta_{z}^{C}-\theta_{\bar{z}}^{C}$ is invariant under factor automorphisms as well.

Definition 4.9 A tuple of non-zero natural numbers $z=\left(n_{1}, \ldots, n_{k}\right)$ is called generic if $\bar{z}$ does not appear as a tuple of $k$ adjacent numbers in $z^{2}=\left(n_{1}, \ldots, n_{k}, n_{1}, \ldots n_{k}\right)$.

Example 4.10 Let $z=\left(n_{1}, \ldots, n_{k}\right)$. If $k \leq 2, z$ is not generic. If $k \geq 3$ and the $n_{i}$ are pairwise distinct, then $z$ is generic. E.g. for $z=(1,2,3)$ we have $\bar{z}=(3,2,1)$ does not appear in $z^{2}=(1,2,3,1,2,3)$.

Proposition 4.11 Let $A * B$ be a free product of two freely indecomposable groups $A$ and $B$, neither of which is infinite cyclic. Then for any generic tuple of natural numbers $z$ the following holds:

1. if $A \nsucceq B$ and $C \in\{A, B\}$ is such that $C ¥ \mathbb{Z} / 2$, then the homogenisation $\bar{f}_{z}^{C}$ of the quasimorphism $f_{z}^{C}$ is an unbounded Aut-invariant quasimorphism on $A * B$;

2. if $A \cong B \nsubseteq \mathbb{Z} / 2$, then the sum $\bar{f}_{z}^{A}+\bar{f}_{z}^{B}$ is an unbounded Aut-invariant quasimorphism on $A * B$.

In both cases the space of homogeneous Aut-invariant quasimorphisms on $A * B$ that vanish on letters has infinite dimension.

Proof First, consider the case $A \nsubseteq B$. Since $A * B$ is not the infinite dihedral group, at least one of the factors is not isomorphic to $\mathbb{Z} / 2$. Without loss of generality we assume $A \supsetneqq \mathbb{Z} / 2$. Let $z$ be generic. By Lemma 4.8 the map $f_{z}^{A}$ defines a quasimorphism invariant under all factor automorphisms. According to Lemma 3.3 this means that $f_{z}^{A}$ is invariant under a full set of representatives for $\operatorname{Out}(A * B)$. Therefore, the homogenisation $\bar{f}_{z}^{A}$ is invariant under all automorphisms of $A * B$ by Lemma 3.2. It remains to check that $\bar{f}_{z}^{A}$ is unbounded, which is equivalent to checking that $f_{z}^{A}$ itself is unbounded by Lemma 2.4.

Since $A \not \mathbb{Z} / 2$, it satisfies $|A| \geq 3$ and we can choose two distinct non-trivial elements $a_{1}, a_{2} \in A$. Furthermore, choose a non-trivial element $b \in B$. Let $z=\left(n_{1}, \ldots, n_{k}\right)$ and choose $m \in \mathbb{N}$ to be non-zero and distinct from all $n_{i} \in \mathbb{N}$. We set

$$
w_{0}=\left(a_{1} b\right)^{n_{1}}\left(a_{2} b\right)^{n_{2}}\left(a_{1} b\right)^{n_{3}}\left(a_{2} b\right)^{n_{4}} \ldots\left(a_{s} b\right)^{n_{k}},
$$

where $s=1$ if $k$ is odd and $s=2$ if $k$ is even. Set

$$
w= \begin{cases}w_{0} & \text { if } k \text { is even, } \\ w_{0}\left(a_{2} b\right)^{m} & \text { if } k \text { is odd. }\end{cases}
$$


The A-code of $w$ is given by

$$
\operatorname{A-code}(w)= \begin{cases}\left(n_{1}, \ldots, n_{k}\right)=z & \text { if } k \text { is even } \\ \left(n_{1}, \ldots, n_{k}, m\right)=(z, m) & \text { if } k \text { is odd }\end{cases}
$$

Since $w$ starts and ends with letters from different groups, the reduced expression of $w^{\ell}$ is the $\ell$-fold product of the word $w$ for all $\ell \in \mathbb{N}$. Moreover, because the first letter from $A$ in $w$ is $a_{1}$ and the last letter from $A$ is $a_{2}$, the A-code of $w^{\ell}$ is

$$
\operatorname{A-code}\left(w^{\ell}\right)= \begin{cases}(z, z, \ldots, z) & \text { if } k \text { is even, } \\ (z, m, z, m, \ldots, z, m) & \text { if } k \text { is odd. }\end{cases}
$$

Since $m$ is distinct from all $n_{i}, m$ can never appear in any occurrence of $z$ or $\bar{z}$ in the A-code of $w^{\ell}$. So $\theta_{z}^{A}\left(w^{\ell}\right)=\ell$, whereas $\theta_{\bar{z}}^{A}\left(w^{\ell}\right)=0$ since $z$ is generic. Consequently,

$$
f_{z}^{A}\left(w^{\ell}\right)=\theta_{z}^{A}\left(w^{\ell}\right)-\theta_{\bar{z}}^{A}\left(w^{\ell}\right)=\ell
$$

which shows that $f_{z}^{A}$ is unbounded.

Second, consider the case $A \cong B$ and fix a choice of isomorphism. Let $z$ be generic. It holds that $|A|=|B| \geq 3$ since $A * B$ is not the infinite dihedral group. Consider the swap isomorphism $s$ interchanging the factors $A$ and $B$, where we use the fixed isomorphism from before to identify $A$ and $B$ with each other. Then the application of $s$ to any element $g$ interchanges the A-code and B-code of $g$ with each other. This implies that the sum $\theta_{z}^{A}+\theta_{z}^{B}$ is invariant under $s$ and consequently the $\operatorname{sum} f_{z}^{A}+f_{z}^{B}$ is invariant under $s$ as well. Again, by Lemma $4.8 f_{z}^{A}$ and $f_{z}^{B}$ define quasimorphisms invariant under all factor automorphisms and so does their sum $f_{z}^{A}+f_{z}^{B}$. According to Lemma 3.3 this means that $f_{z}^{A}+f_{z}^{B}$ is invariant under a full set of representatives for $\operatorname{Out}(A * B)$. Again, by Lemma 3.2 we see that the homogenisation $\bar{f}_{z}^{A}+\bar{f}_{z}^{B}$ is invariant under all automorphisms of $A * B$. It remains to verify unboundedness.

For this let $a_{1}, a_{2} \in A$ and $b_{1}, b_{2} \in B$ be non-trivial such that $a_{1} \neq a_{2}$ and $b_{1} \neq b_{2}$. Pick a non-zero number $m \in \mathbb{N}$ distinct from all $n_{i} \in \mathbb{N}$, where $z=\left(n_{1}, \ldots, n_{k}\right)$. As before, we set

$$
w_{0}=\left(a_{1} b_{1}\right)^{n_{1}}\left(a_{2} b_{2}\right)^{n_{2}}\left(a_{1} b_{1}\right)^{n_{3}}\left(a_{2} b_{2}\right)^{n_{4}} \ldots\left(a_{s} b_{s}\right)^{n_{k}},
$$

where $s$ is 1 or 2 depending on whether $k$ is odd or even. We set

$$
w= \begin{cases}w_{0} & \text { if } k \text { is even } \\ w_{0}\left(a_{2} b_{2}\right)^{m} & \text { if } k \text { is odd. }\end{cases}
$$

Then the A-code and B-code of $w$ agree and are given by

$$
\mathrm{A}-\operatorname{code}(w)=\mathrm{B}-\operatorname{code}(w)= \begin{cases}\left(n_{1}, \ldots, n_{k}\right)=z & \text { if } k \text { is even } \\ \left(n_{1}, \ldots, n_{k}, m\right)=(z, m) & \text { if } k \text { is odd }\end{cases}
$$

Since $m$ is distinct from all $n_{i}, m$ can never appear in any occurrence of $z$ or $\bar{z}$ in the A-code and B-code of $w^{\ell}$. As in the first case, $\theta_{z}^{A}\left(w^{\ell}\right)=\theta_{z}^{B}\left(w^{\ell}\right)=\ell$, whereas $\theta_{\bar{z}}^{A}\left(w^{\ell}\right)=$ $\theta_{\bar{z}}^{B}\left(w^{\ell}\right)=0$ since $z$ is generic. Consequently,

$$
f_{z}^{A}\left(w^{\ell}\right)+f_{z}^{B}\left(w^{\ell}\right)=\theta_{z}^{A}\left(w^{\ell}\right)+\theta_{z}^{B}\left(w^{\ell}\right)-\theta_{\bar{z}}^{A}\left(w^{\ell}\right)-\theta_{\bar{z}}^{B}\left(w^{\ell}\right)=2 \ell,
$$

which shows that $f_{z}^{A}+f_{z}^{B}$ is unbounded and therefore its homogenisation is the desired unbounded Aut-invariant quasimorphism on $A * B$. 
Finally, let us verify that the space of homogeneous Aut-invariant quasimorphisms on $A * B$ that vanish on letters is infinite-dimensional. Let $r \in \mathbb{N}$ and let $z_{1}, \ldots, z_{r}$ be generic tuples. Choose $z_{r+1}$ be a 3 -tuple whose entries are distinct non-zero natural numbers and do not appear in any of the $z_{i}$; then $z_{r+1}$ is generic. It follows from the above construction of the word $w$ for $z_{r+1}$ in both cases that any linear combination of the associated quasimorphisms $f_{z_{1}}^{A}+f_{z_{1}}^{B}, \ldots, f_{z_{r}}^{A}+f_{z_{r}}^{B}$ vanishes on all powers of $w$. It follows that the same holds for any linear combination of their homogenisations $\bar{f}_{z_{1}}^{A}+\bar{f}_{z_{1}}^{B}, \ldots, \bar{f}_{z_{r}}^{A}+\bar{f}_{z_{r}}^{B}$. Thus, $\bar{f}_{z_{r+1}}^{A}+\bar{f}_{z_{r 1}}^{B}$ is not contained in the subspace spanned by the first $r$ quasimorphisms. Clearly, the homogenisation of any code quasimorphism vanishes on all letters of $A * B$. Since $r \in \mathbb{N}$ was arbitrary, it follows that the space of homogeneous Aut-invariant quasimorphisms on $A * B$ that vanish on letters cannot have finite dimension.

\section{Weighted code quasimorphisms}

If one of the factors of a free product $A * B$ of freely indecomposable groups happens to be infinite cyclic, the code quasimorphisms above are in general not Aut-invariant since they are not necessarily invariant with respect to transvections. Thus, we need to modify our original construction to deal with infinite cyclic factors. Afterwards we will follow steps similar to the previous section in order to establish their Aut-invariance.

Lemma 5.1 Let $B$ be a non-trivial group and let $w$ be any word in $\mathbb{Z} * B$ such that $w$ only contains letters of the same sign from $\mathbb{Z}$ and starts and ends with a non-zero letter from $\mathbb{Z}$. Then its unique reduced form $w^{\prime}$ starts and ends with a letter from $\mathbb{Z}$ with that given sign. Moreover, the sum over all letters in $w$ belonging to the factor $\mathbb{Z}$ remains the same in its reduced form $w^{\prime}$.

Proof Any word in the free product is brought to its reduced form by successively eliminating trivial letters and replacing two adjacent letters from the same factor by their product in that factor. The sum of all letters from $\mathbb{Z}$ stays the same because any two adjacent letters of $\mathbb{Z}$ are always replaced by their sum throughout the reduction process. The only way to encounter an elimination of the first letter $a_{1} \in \mathbb{Z}$ or the last letter $a_{n} \in \mathbb{Z}$ during the reduction process would be by the occurrence of $-a_{1}$ or $-a_{n}$. This is not possible since $a_{1}$ and $a_{n}$ are non-zero and all letters have the same sign by assumption.

Definition 5.2 (Weighted $\mathbb{Z}$-code) Let $B$ be freely indecomposable and $B \nsubseteq \mathbb{Z}$. Write $g \in \mathbb{Z} * B$ in reduced form. Let $\left(a_{1}, \ldots, a_{k}\right)$ be the $\mathbb{Z}$-tuple of $g$. We define a tuple $\left(x_{1}, \ldots, x_{\ell}\right)$ of non-zero natural numbers as follows. Consider the successive subsequences of maximal length in $\left(a_{1}, \ldots, a_{k}\right)$ consisting of integers all of the same sign. For the $i$-th such sequence, we define $x_{i}$ to be the absolute value of the sum of integers in that sequence. We call the tuple $\left(x_{1}, \ldots, x_{\ell}\right)$ the weighted $\mathbb{Z}$-code of $g$.

Example 5.3 Let $B$ be a non-trivial group and let $b_{i} \in B$ be non-trivial elements. Then the reduced word

$$
w=7 b_{1}(-2) b_{2}(-4) b_{3}(-1) b_{4} 9 b_{5} 2 b_{6}(-3)
$$

has the $\mathbb{Z}$-tuple $(7,-2,-4,-1,9,2,-3)$ which yields the weighted $\mathbb{Z}$-code $(7,7,11,3)$.

Definition 5.4 (Weighted code quasimorphisms) Let $z=\left(n_{1}, \ldots, n_{k}\right)$ be a tuple of non-zero natural numbers. We set $\theta_{z}^{\mathbb{Z}}: \mathbb{Z} * B \rightarrow \mathbb{Z}_{\geq 0}$ to count the number of disjoint appearances of 
$z$ as a tuple of consecutive numbers inside the weighted $\mathbb{Z}$-code of $g \in \mathbb{Z} * B$. Define the weighted code quasimorphism

$$
f_{z}^{\mathbb{Z}}: \mathbb{Z} * B \rightarrow \mathbb{Z} \quad \text { by } \quad f_{z}^{\mathbb{Z}}(g)=\theta_{z}^{\mathbb{Z}}(g)-\theta_{z}^{\mathbb{Z}}\left(g^{-1}\right)
$$

for all $g \in \mathbb{Z} * B$. Note that we again have $\theta_{z}^{\mathbb{Z}}\left(g^{-1}\right)=\theta_{\bar{z}}^{\mathbb{Z}}(g)$ for all $g$.

Lemma 5.5 Let $z$ be a non-empty tuple of non-zero natural numbers. Then the counting function $\theta_{z}^{\mathbb{Z}}: \mathbb{Z} * B \rightarrow \mathbb{Z}_{\geq 0}$ satisfies

1. $\theta_{z}^{\mathbb{Z}}\left(g^{-1}\right)=\theta_{\bar{z}}^{\mathbb{Z}}(g)$ for all $g \in \mathbb{Z} * B$,

2. $\left|\theta_{z}^{\mathbb{Z}}\left(w_{1} w_{2}\right)-\theta_{z}^{\mathbb{Z}}\left(w_{1}\right)-\theta_{z}^{\mathbb{Z}}\left(w_{2}\right)\right| \leq 2$ for all reduced words $w_{1}$, w2 in $\mathbb{Z} * B$ for which $w_{1} w_{2}$ is a reduced word.

Moreover, $f_{z}^{\mathbb{Z}}: \mathbb{Z} * B \rightarrow \mathbb{Z}$ defined for all $g \in \mathbb{Z} * B$ by $f_{z}^{\mathbb{Z}}(g)=\theta_{z}^{\mathbb{Z}}(g)-\theta_{z}^{\mathbb{Z}}\left(g^{-1}\right)$ is a quasimorphism of defect $D\left(f_{z}^{\mathbb{Z}}\right) \leq 30$.

Proof First, recall that the reduced form of $g^{-1}$ is obtained by inverting the reduced form of $g$, which amounts to reversing the order and inverting all letters. This means to obtain the weighted $\mathbb{Z}$-code of $g^{-1}$ one needs to reverse the one of $g$. Consequently, counting the number of disjoint occurrences of $z$ in the weighted $\mathbb{Z}$-code of $g^{-1}$ amounts to counting the disjoint occurrences of the reversed tuple $\bar{z}$ in the weighted $\mathbb{Z}$-code of $g$ itself. This proves the first part.

Second, let $w_{1}, w_{2}$ be written as reduced words with $\mathbb{Z}$-tuples given by $\left(n_{1}, \ldots, n_{k}\right)$ for $w_{1}$ and $\left(m_{1}, \ldots, m_{\ell}\right)$ for $w_{2}$ for integers $n_{i}, m_{j}$. Let $\left(x_{1}, \ldots, x_{k^{\prime}}\right)$ and $\left(y_{1}, \ldots, y_{\ell^{\prime}}\right)$ be the weighted $\mathbb{Z}$-codes of $w_{1}$ and $w_{2}$. By assumption there is no cancellation or reduction in the product of their reduced expressions representing $w_{1} w_{2}$. That means that the last letter of $w_{1}$ and the first letter of $w_{2}$ belong to different factors. Then

weighted $\mathbb{Z}$-code $\left(w_{1} w_{2}\right)= \begin{cases}\left(x_{1}, \ldots, x_{k^{\prime}}, y_{1}, \ldots, y_{\ell^{\prime}}\right) & \text { if } \operatorname{sgn}\left(n_{k}\right) \neq \operatorname{sgn}\left(m_{1}\right), \\ \left(x_{1}, \ldots, x_{k^{\prime}-1}, x_{k^{\prime}}+y_{1}, y_{2}, \ldots, y_{\ell^{\prime}}\right) & \text { if } \operatorname{sgn}\left(n_{k}\right)=\operatorname{sgn}\left(m_{1}\right) .\end{cases}$

If $\operatorname{sgn}\left(n_{k}\right) \neq \operatorname{sgn}\left(m_{1}\right)$, then $\theta_{z}^{\mathbb{Z}}\left(w_{1} w_{2}\right) \in\left\{\theta_{z}^{\mathbb{Z}}\left(w_{1}\right)+\theta_{z}^{\mathbb{Z}}\left(w_{2}\right), \theta_{z}^{\mathbb{Z}}\left(w_{1}\right)+\theta_{z}^{\mathbb{Z}}\left(w_{2}\right)+1\right\}$ since at most one of the disjoint occurrences of $z$ can involve numbers that do not lie completely in the weighted $\mathbb{Z}$-code of either $w_{1}$ or $w_{2}$.

If $\operatorname{sgn}\left(n_{k}\right)=\operatorname{sgn}\left(m_{1}\right)$, then $\theta_{z}^{\mathbb{Z}}\left(w_{1} w_{2}\right) \geq \theta_{z}^{\mathbb{Z}}\left(w_{1}\right)+\theta_{z}^{\mathbb{Z}}\left(w_{2}\right)-2$ since only one occurrence of $z$ in the weighted $\mathbb{Z}$-code of $w_{1}$ and $w_{2}$ can involve the first or last number respectively. Moreover, if an occurrence of $z$ in the weighted $\mathbb{Z}$-code of $w_{1} w_{2}$ involves $x_{k^{\prime}}+y_{1}$, then all other occurrences are fully contained in the weighted $\mathbb{Z}$-code of either $w_{1}$ or $w_{2}$. Thus, $\theta_{z}^{\mathbb{Z}}\left(w_{1} w_{2}\right) \leq \theta_{z}^{\mathbb{Z}}\left(w_{1}\right)+\theta_{z}^{\mathbb{Z}}\left(w_{2}\right)+1$.

In both cases we conclude that

$$
\left|\theta_{z}^{\mathbb{Z}}\left(w_{1} w_{2}\right)-\theta_{z}^{\mathbb{Z}}\left(w_{1}\right)-\theta_{z}^{C}\left(w_{2}\right)\right| \leq 2 .
$$

It follows from Lemma 2.12 that $f_{z}^{\mathbb{Z}}$ is a quasimorphism of defect at most 30 .

Lemma 5.6 For all non-empty tuples $z$ the weighted code quasimorphism $f_{z}^{\mathbb{Z}}: \mathbb{Z} * B \rightarrow \mathbb{Z}$ is invariant under factor automorphisms and transvections.

Proof It is immediate from the definition that the weighted $\mathbb{Z}$-code of any element in the free product is invariant under factor automorphisms. Let $x$ be a generator of the infinite cyclic factor in $\mathbb{Z} * B$. Any transvection is defined to be the identity on letters from $B$ and maps 
$x \rightarrow x y$ or $x \rightarrow y x$ for some non-trivial element $y \in B$. Let us consider the transvection $\varphi$ uniquely specified by $x \rightarrow x y$ and show that the weighted $\mathbb{Z}$-code of any element in $\mathbb{Z} * B$ is invariant under $\varphi$. Then it immediately follows that $\theta_{z}^{\mathbb{Z}}$ and $f_{z}^{\mathbb{Z}}$ are invariant under $\varphi$. The argument for transvections of the second kind will follow analogously to the one we present now.

Let $w \in \mathbb{Z} * B$ be a reduced word such that its weighted $\mathbb{Z}$-code has length one. This means that all letters from $\mathbb{Z}$ in the reduced expression of $w$ have the same sign and the weighted $\mathbb{Z}$-code is given by the image of $w$ under the factor projection $\mathbb{Z} * B \rightarrow \mathbb{Z}$. Note that this factor projection is invariant with respect to $\varphi$ and so the weighted $\mathbb{Z}$-code of $\varphi(w)$ agrees with the one of $w$. There cannot be any cancellations of letters from $\mathbb{Z}$ occurring.

Let us do a preliminary calculation to visualise the general case more easily. Let $k, \ell$ be non-zero natural numbers and $b \in B$ non-trivial. Then

$$
\begin{aligned}
& \varphi\left(x^{k} b x^{-\ell}\right)=\varphi(x)^{k} b \varphi(x)^{-\ell}=(x y)^{k} b\left(y^{-1} x^{-1}\right)^{\ell}=x y \ldots x y x y b y^{-1} x^{-1} y^{-1} x^{-1} \ldots y^{-1} x^{-1}, \\
& \varphi\left(x^{-k} b x^{\ell}\right)=\varphi(x)^{-k} b \varphi(x)^{\ell}=\left(y^{-1} x^{-1}\right)^{k} b(x y)^{\ell}=y^{-1} x^{-1} \ldots y^{-1} x^{-1} b x y \ldots x y .
\end{aligned}
$$

This shows that the letter from $B$ separating the positive and negative powers of $x$ either remains $b$ or is a conjugate of $b$ in $B$ after applying $\varphi$.

Let $w \in \mathbb{Z} * B$ be a reduced word with weighted $\mathbb{Z}$-code of length $k \geq 2$. In $w$ we formally gather all consecutive occurrences of powers of $x$ of the same sign and call these sub-words $w_{i}$ for $i=\{1, \ldots, k\}$. That is, we write the reduced word $w$ uniquely as a product of reduced words as

$$
w=w_{1} b_{1} w_{2} b_{2} \ldots w_{k-1} b_{k-1} w_{k}
$$

where the $b_{i} \in B$ are non-trivial and the $w_{i}$ are of of maximal length such that all letters from $\mathbb{Z}$ inside any $w_{i}$ have the same sign. Moreover, in this decomposition $w_{1}$ ends with a letter from $\mathbb{Z}, w_{n}$ starts with a letter from $\mathbb{Z}$ and all other $w_{i}$ start and end with letters from $\mathbb{Z}$. By the maximality of $w_{i}$ all letters from $\mathbb{Z}$ occurring in $w_{i}$ have different signs from the ones occurring in $w_{i+1}$ for all $i$.

We apply $\varphi$ to $w$ and obtain an a priori not necessarily reduced word, which we rewrite in the previous block form as

$$
\varphi(w)=\varphi\left(w_{1}\right) b_{1} \varphi\left(w_{2}\right) b_{2} \ldots \varphi\left(w_{k-1}\right) b_{k-1} \varphi\left(w_{k}\right)=w_{1}^{\prime} b_{1}^{\prime} w_{2}^{\prime} b_{2}^{\prime} \ldots w_{k-1}^{\prime} b_{k-1}^{\prime} w_{k}^{\prime},
$$

where $b_{i}^{\prime}=y b y^{-1}$ if the letters from $\mathbb{Z}$ change sign from positive to negative at $b_{i}$ and $b_{i}^{\prime}=b_{i}$ if they change from negative to positive. Moreover, all letters from $\mathbb{Z}$ inside any $w_{i}^{\prime}$ have the same sign again, $w_{1}^{\prime}$ ends with a letter from $\mathbb{Z}, w_{n}^{\prime}$ starts with a letter from $\mathbb{Z}$ and all other $w_{i}^{\prime}$ start and end with letters from $\mathbb{Z}$.

We observe that when bringing $\varphi(w)$ to its reduced form there cannot be any cancellations of the letters $b_{i}^{\prime}$. This is because by Lemma 5.1 the letters that are adjacent to $b_{i}$ will always remain letters from $\mathbb{Z}$ after the reduction procedure of all $w_{i}^{\prime}$. Indeed, replacing all $w_{i}^{\prime}$ by their reduced forms $w_{i}^{\prime \prime}$ we see that the product

$$
w^{\prime \prime}=w_{1}^{\prime \prime} b_{1}^{\prime} w_{2}^{\prime \prime} b_{2} \ldots w_{k-1}^{\prime \prime} b_{k-1}^{\prime} w_{k}^{\prime \prime}
$$

is the reduced representative of $\varphi(w)$ since the letters adjacent to the $b_{i}^{\prime}$ are always letters from $\mathbb{Z}$. Consequently, no cancellations in between letters of different signs from $\mathbb{Z}$ can occur when bringing $\varphi(w)$ to its reduced form. The reduced words $w_{i}^{\prime \prime}$ have the same weighted $\mathbb{Z}$-code as the original $w_{i}$ for all $i$. Therefore, the weighted $\mathbb{Z}$-code of $\varphi(w)$ agrees with the weighted $\mathbb{Z}$-code of $w$. 
Proposition 5.7 Let $B$ be a freely indecomposable group which is not infinite cyclic. Then for any generic tuple of natural numbers $z$ the homogenisation $\bar{f}_{z}^{\mathbb{Z}}: \mathbb{Z} * B \rightarrow \mathbb{R}$ of the quasimorphism $f_{z}^{\mathbb{Z}}$ is an unbounded Aut-invariant quasimorphism on $\mathbb{Z} * B$. Moreover, the space of homogeneous Aut-invariant quasimorphisms on $\mathbb{Z} * B$ that vanish on letters has infinite dimension.

Proof By Lemma 5.5 $f_{z}^{\mathbb{Z}}$ is a quasimorphism, which is invariant under factor automorphisms and transvections according to Lemma 5.6. Images of these automorphisms generate the outer automorphism group Out $(\mathbb{Z} * B)$ by Lemma 3.3. Thus, $f_{z}^{\mathbb{Z}}$ is invariant under a full set of representatives of all outer automorphisms and so by Lemma 3.2 the homogenisation $\bar{f}_{z}^{\mathbb{Z}}$ is invariant under $\operatorname{Aut}(\mathbb{Z} * B)$. It remains to check that it is unbounded, which is equivalent to $f_{z}^{\mathbb{Z}}$ itself being unbounded.

Since $z$ is generic, $z=\left(n_{1}, \ldots, n_{k}\right)$ for some $k \geq 3$ where all $n_{i} \in \mathbb{N}$ are non-zero. Let $b \in B$ be non-trivial and $m$ a strictly positive integer number distinct from all $n_{i}$. Set $w \in \mathbb{Z} * B$ to be

$$
w= \begin{cases}n_{1} b\left(-n_{2}\right) b n_{3} b\left(-n_{4}\right) \ldots b\left(-n_{k}\right) b & \text { if } k \text { is even } \\ n_{1} b\left(-n_{2}\right) b n_{3} b\left(-n_{4}\right) \ldots b\left(-n_{k}\right) b m b & \text { if } k \text { is odd }\end{cases}
$$

The weighted $\mathbb{Z}$-code of $w$ is given by

$$
\text { weighted } \mathbb{Z} \text {-code }(w)= \begin{cases}\left(n_{1}, \ldots, n_{k}\right)=z & \text { if } k \text { is even, } \\ \left(n_{1}, \ldots, n_{k}, m\right)=(z, m) & \text { if } k \text { is odd }\end{cases}
$$

Since $w$ starts and ends with letters belonging to different factors, the reduced expression of $w^{\ell}$ is the $\ell$-fold product of the word $w$ for all $\ell \in \mathbb{N}$. Moreover, since the first and last letter from $\mathbb{Z}$ in $w$ have different signs the weighted $\mathbb{Z}$-code of $w^{\ell}$ is

$$
\text { weighted } \mathbb{Z} \text {-code }\left(w^{\ell}\right)= \begin{cases}(z, z, \ldots, z) & \text { if } k \text { is even, } \\ (z, m, z, m, \ldots, z, m) & \text { if } k \text { is odd. }\end{cases}
$$

Since $m$ is distinct from all $n_{i}, m$ cannot appear in any occurrence of $z$ or $\bar{z}$ inside the weighted $\mathbb{Z}$-code of $w^{\ell}$. So $\theta_{z}^{\mathbb{Z}}\left(w^{\ell}\right)=\ell$, whereas $\theta_{\bar{z}}^{A}\left(w^{\ell}\right)=0$ since $z$ is generic. Consequently,

$$
f_{z}^{\mathbb{Z}}\left(w^{\ell}\right)=\theta_{z}^{\mathbb{Z}}\left(w^{\ell}\right)-\theta_{\bar{z}}^{\mathbb{Z}}\left(w^{\ell}\right)=\ell,
$$

which shows that $f_{z}^{\mathbb{Z}}$ is unbounded.

Finally, let us verify that the space of homogeneous Aut-invariant quasimorphisms on $\mathbb{Z} * B$ that vanish on letters is infinite-dimensional. Let $r \in \mathbb{N}$ and let $z_{1}, \ldots, z_{r}$ be generic tuples. Choose $z_{r+1}$ be a 3-tuple whose entries are distinct non-zero natural numbers and do not appear in any of the $z_{i}$; then $z_{r+1}$ is generic. It follows from the above construction of the word $w$ for $z_{r+1}$ that any linear combination of $f_{z_{1}}^{\mathbb{Z}}, \ldots, f_{z_{r}}^{\mathbb{Z}}$ vanishes on all powers of this $w$. It follows that the same holds for any linear combination of their homogenisations $\bar{f}_{z_{1}}^{\mathbb{Z}}, \ldots, \bar{f}_{z_{r}}^{\mathbb{Z}}$. Thus, $\bar{f}_{z_{r+1}}^{\mathbb{Z}}$ is not contained in the subspace spanned by the first $r$ quasimorphisms. Clearly, the homogenisation of any weighted code quasimorphism vanishes on all letters of $\mathbb{Z} * B$. Since, $r \in \mathbb{N}$ was arbitrary, it follows that the space of homogeneous Aut-invariant quasimorphisms on $\mathbb{Z} * B$ that vanish on letters cannot have finite dimension.

Remark 5.8 Proposition 5.7 does not hold for $B=\mathbb{Z}$. Indeed, $\bar{f}_{z}^{\mathbb{Z}}$ does no longer need to be invariant under $\operatorname{Aut}(\mathbb{Z} * B)$ since the weighted $\mathbb{Z}$-code is in general not invariant under transvections of the factor $B$. 
For example, consider $z=(4,3,2,1)$ and denote the standard generators of $\mathbb{Z} * B$ by $x \in \mathbb{Z}$ and $y \in B$. Following the proof of Proposition 5.7 the element $w=x^{4} y x^{-3} y x^{2} y x^{-1} y$ with weighted $\mathbb{Z}$-code of $(4,3,2,1)$ satisfies $f_{z}^{\mathbb{Z}}\left(w^{\ell}\right)=\ell$ for all $\ell \in \mathbb{N}$. Let $\varphi \in \operatorname{Aut}(\mathbb{Z} * B)$ be the transvection of the second factor defined by $\varphi(x)=x$ and $\varphi(y)=x^{3} y$. Then $\varphi(w)=x^{7} y^{2} x^{5} y x^{2} y$. So the weighted $\mathbb{Z}$-code of $\varphi(w)$ is the tuple with a single entry equal to 14 . Thus, $f_{z}^{\mathbb{Z}}\left(\varphi\left(w^{\ell}\right)\right)=f_{z}^{\mathbb{Z}}\left(\varphi(w)^{\ell}\right)=0$ for all $\ell \in \mathbb{N}$. So $\bar{f}_{z}^{\mathbb{Z}}$ evaluates non-trivially on $w$, but trivially on $\varphi(w)$, which means that $\bar{f}_{z}^{\mathbb{Z}}$ is not invariant under $\operatorname{Aut}(\mathbb{Z} * B)$.

Moreover, this example can be used to show that the sum of the two weighted code quasimorphisms $\bar{f}_{z}^{\mathbb{Z}}+\bar{f}_{z}^{B}$ is not necessarily invariant under Aut $(\mathbb{Z} * B)$ either, which contrasts the situation of free factors that are not infinite cyclic in Propostion 4.11 (2).

\section{Applications of code quasimorphisms}

Proof of Theorem 1 By [3, Theorem 2] the space of homogeneous Aut-invariant quasimorphisms on $\mathbb{Z} * \mathbb{Z}$ is infinite-dimensional. Inverting both generators of the factors defines an automorphism which inverts all letters in $\mathbb{Z} * \mathbb{Z}$. So any homogeneous Aut-invariant quasimorphism on $\mathbb{Z} * \mathbb{Z}$ vanishes on all letters. For all other free products of two factors Proposition 4.11 and Proposition 5.7 imply the existence of infinitely many linearly independent homogeneous Aut-invariant quasimorphisms, all of which vanish on letters.

Proof of Corollary 1.1 Let $A * B$ be a free product of two freely indecomposable groups which is not the infinite dihedral group. By Theorem 1 there exist unbounded Aut-invariant quasimorphisms on $A * B$ that are bounded on all letters. Since $A * B$ is generated by letters, the result follows from Lemma 2.7 .

Remark 6.1 If neither $A$ nor $B$ is infinite cyclic, then Corollary 1.1 can also be deduced from the result given in [13, Lemma 4.4] together with the explicit description of the automorphism group given in Sect. 3 .

Corollary 6.2 Let $G=*_{i \in I} G_{i}$ be a free product of finitely many freely indecomposable groups $G_{i}$. Assume there exist free factors $G_{j}$ and $G_{k}$ with $j \neq k$ such that no free factor $G_{i}$ for $i \notin\{j, k\}$ is isomorphic to $G_{j}$ or $G_{k}$ or is infinite cyclic. Moreover, assume that $G_{j}, G_{k}$ are not both equal to $\mathbb{Z} / 2$. Then any unbounded Aut-invariant quasimorphism on $G_{j} * G_{k}$ gives rise to an unbounded Aut-invariant quasimorphism on G. In particular, the space of homogeneous Aut-invariant quasimorphisms on $G$ is infinite-dimensional.

Proof We claim that the projection $p: G \rightarrow G_{j} * G_{k}$ is Aut-equivariant, i.e. any automorphism of $G$ descends via $p$ to an automorphism of $G_{j} * G_{k}$. This is equivalent to $\operatorname{ker}(p)$ being a characteristic subgroup of $G$. Once this is established, we apply Theorem 1 to $G_{j} * G_{k}$ and conclude the proof by applying Lemma 3.4.

Let us now show that any automorphism of $G$ indeed descends to $G_{j} * G_{k}$. By [8] $\operatorname{Aut}(G)$ is generated by factor automorphisms, swap automorphisms, partial conjugations and transvections since inner automorphisms can be written as products of factor automorphisms and partial conjugations. It is clear that all factor automorphisms and all partial conjugations of $G$ descend to automorphisms of $G_{j} * G_{k}$ via $p$. By our assumption there are no swap automorphisms permuting any other free factors in $G$ with $G_{j}$ and $G_{k}$, so these descend to the quotient as well. It only remains to check the transvections if $G_{j}$ or $G_{k}$ happen to be infinite cyclic. So let $G_{j}$ be infinite cyclic generated by $x$ and let $a$ be a letter from a different factor $G_{\ell}$. If $\ell=k$, then any transvection $\varphi_{a}$ defined by $\varphi_{a}(x)=a x$ or $\varphi_{a}(x)=x a$ descends 
via $p$ to the same transvection on $G_{j} * G_{k}$. If $\ell \neq k$, any such transvection descends to the identity on $G_{j} * G_{k}$. In particular, it always descends via $p$. Since a generating set of $\operatorname{Aut}(G)$ descends to automorphisms of the quotient $G_{j} * G_{k}$, any element of $\operatorname{Aut}(G)$ does so. Consequently, the map $p$ is Aut-equivariant.

Corollary 6.3 Let $H \rightarrow G \rightarrow A * B$ be an extension of a free product offreely indecomposable groups $A$ and $B$ by a group $H$. Assume that $H$ is a characteristic subgroup of $G$ and $A * B$ is not the infinite dihedral group. Then the space of homogeneous Aut-invariant quasimorphisms on $G$ is infinite-dimensional.

Proof The space of homogeneous Aut-invariant quasimorphisms on $A * B$ is infinitedimensional by Theorem 1. Therefore, the result follows from Lemma 3.4.

Corollary 6.4 Let $G_{1} *_{H} G_{2}$ be a free product of groups $G_{1}, G_{2}$ amalgamated over a common subgroup $H$ which is proper and central in both $G_{1}$ and $G_{2}$. If $G_{1} / H$ and $G_{2} / H$ are freely indecomposable and not both equal to $\mathbb{Z} / 2$, the space of homogeneous Aut-invariant quasimorphisms on $G_{1} *_{H} G_{2}$ is infinite-dimensional.

Proof By assumption $H \neq G_{1}$ and $H \neq G_{2}$ and so $H$ equals the center of $G_{1} *_{H} G_{2}$. As such it is a characteristic subgroup of $G_{1} *_{H} G_{2}$. Furthermore, $\frac{G_{1} *_{H} G_{2}}{H} \cong \frac{G_{1}}{H} * \frac{G_{2}}{H}$. By assumption $\frac{G_{1}}{H} * \frac{G_{2}}{H}$ is not isomorphic to the infinite dihedral group and the result follows from Corollary 6.3 above.

Example 6.5 For $q \geq 3$, the Hecke groups $H_{q} \cong \mathbb{Z} / 2 * \mathbb{Z} / q$ admit infinitely many linearly independent homogeneous Aut-invariant quasimorphisms by Theorem 1.

Example 6.6 By Corollary 6.4 the space of homogeneous Aut-invariant quasimorphisms on $\operatorname{SL}(2, \mathbb{Z})$ is infinite-dimensional, since $\operatorname{SL}(2, \mathbb{Z})$ is the amalgamated product $\mathbb{Z} / 4 * \mathbb{Z} / 2 \mathbb{Z} / 6$.

Example 6.7 The braid group $B_{3}$ admits infinitely many linearly independent homogeneous Aut-invariant quasimorphism as well by Corollary 6.3. Indeed, $B_{3}$ is the universal central extension of $\operatorname{PSL}(2, \mathbb{Z})=\mathbb{Z} / 2 * \mathbb{Z} / 3$ by $\mathbb{Z}$.

Example 6.8 Let $G_{p, q}=\mathbb{Z} * \mathbb{Z} \mathbb{Z}$ be the free product of two copies of the integers amalgamated over inclusions $\iota_{1}, \iota_{2}: \mathbb{Z} \rightarrow \mathbb{Z}$ which are multiplication by $p$ and $q$. For coprime choices of $p$ and $q$ these are the so called knot groups $K_{p, q}$ arising as the fundamental group of the complement of torus knots. Then $G_{p, q}$ admits infinitely many linearly independent homogeneous Aut-invariant quasimorphisms if $\min \{|p|,|q|\} \geq 2$ and $\max \{|p|,|q|\} \geq 3$. We have seen in Example 2.10 that this is no longer true for $p=q=2$.

Example 6.9 $B_{3} *_{\mathbb{Z}} B_{3}$, the free product of $B_{3}$ with itself amalgamated over their common center generated by the Garside element, admits infinitely many linearly independent unbounded Aut-invariant quasimorphisms. To prove this we cannot apply Corollary 6.4 directly since $B_{3} / \mathbb{Z}$ is not freely indecomposable. The center of $B_{3} * \mathbb{Z} B_{3}$ is again generated by the Garside element of each of the factors. This fits into the short exact sequence

$$
\mathbb{Z} \rightarrow B_{3} * \mathbb{Z} B_{3} \rightarrow\left(B_{3} / \mathbb{Z}\right) *\left(B_{3} / \mathbb{Z}\right) .
$$

Finally, $\left(B_{3} / \mathbb{Z}\right) *\left(B_{3} / \mathbb{Z}\right)=\operatorname{PSL}(2, \mathbb{Z}) * \operatorname{PSL}(2, \mathbb{Z})=\mathbb{Z} / 2 * \mathbb{Z} / 3 * \mathbb{Z} / 2 * \mathbb{Z} / 3$. So Corollary 6.2 applies with $G_{j}=G_{k}=\mathbb{Z} / 3$. Then the statement for $B_{3} * \mathbb{Z} B_{3}$ follows from Lemma 3.4 . 


\section{Aut-invariant stable commutator length}

For any group $G$ let $\mathrm{cl}_{G}$ denote the commutator length on $[G, G]$, which is defined to be the minimal number of commutators required to write a given element of the commutator subgroup. Let $\operatorname{scl}_{G}(x)=\lim _{n} \frac{\operatorname{cl}\left(x^{n}\right)}{n}$ denote the stable commutator length of $x \in[G, G]$. It shares a deep relationship with quasimorphisms on $G$ through the so called Bavard duality [6]. We now define the Aut-invariant (stable) commutator length; this is a special case of the so called $\hat{G}$-invariant (stable) commutator length defined in [12] for groups $\hat{G}$ in which $G$ is a normal subgroup.

Definition 7.1 Let $G \leq \hat{G}$ be a normal subgroup. Consider the subgroup $[\hat{G}, G] \leq G$ generated by commutators of the form $[F, g]$ and their inverses where $F \in \hat{G}$ and $g \in G$. Then for $x \in[\hat{G}, G]$ the $\hat{G}$-invariant commutator length $\mathrm{cl}_{\hat{G}, G}(x)$ is defined to be the minimal length of an expression of $x$ as a product of commutators $[F, g]$ and their inverses where $F \in \hat{G}$ and $g \in G$. The $\hat{G}$-invariant stable commutator length $\operatorname{scl}_{\hat{G}, G}$ for $x \in[\hat{G}, G]$ is defined by $\operatorname{scl}_{\hat{G}, G}(x)=\lim _{n} \frac{\mathrm{cl}_{\hat{G} G}\left(x^{n}\right)}{n}$.

Given any group $G$, its inner automorphism group $\operatorname{Inn}(G)$ is a normal subgroup of $\operatorname{Aut}(G)$ and so the above definition applies to $\operatorname{Inn}(G)$. If $G$ has trivial center, $G$ can be identified with $\operatorname{Inn}(G)$. In this case we simplify the notation by denoting the Aut $(G)$-invariant commutator length simply as $\mathrm{cl}_{\mathrm{Aut}}$ and the $\mathrm{Aut}(G)$-invariant stable commutator length simply as $\mathrm{scl}_{\mathrm{Aut}}$.

Setting $\hat{G}=\operatorname{Aut}(G)$ the following lemma is proven in [12, Lemma 2.1].

Lemma 7.2 Let $G$ be a group with trivial center so that $G=\operatorname{Inn}(G)$. Let $\phi$ be an homogeneous Aut-invariant quasimorphism on $G$. Then any $x \in[\operatorname{Aut}(G), G] \leq G$ satisfies

$$
\operatorname{scl}_{\text {Aut }}(x) \geq \frac{1}{2} \frac{|\phi(x)|}{D(\phi)} .
$$

In fact, according to [12, Theorem 1.3] $\hat{G}$-invariant quasimorphisms satisfy an analogue of the Bavard duality theorem if $[\hat{G}, G]=G$. All free products $A * B$ of freely indecomposable groups $A$ and $B$ have trivial center and so the notions $\mathrm{cl}_{\text {Aut }}$ and $\mathrm{scl}_{\text {Aut }}$ apply. However, free products often fail to satisfy $[\operatorname{Aut}(G), G]=G$. We will use a constructive approach rather than relying on an invariant analogue of Bavard's duality in the following.

Example 7.3 For $D_{\infty}=\mathbb{Z} / 2 * \mathbb{Z} / 2$ it holds that $\mathrm{scl}_{\text {Aut }} \equiv 0$. To see this denote the generators of the $\mathbb{Z} / 2$ factors by $a$ and $b$ and let $s$ be the automorphism of $D_{\infty}$ interchanging $a$ and $b$. Then we calculate $[s, a]=s a s^{-1} a^{-1}=s(a) a^{-1}=b a$ and $[s, b]=a b=(b a)^{-1}$. Since the total number of letters appearing in any expression of the form $[f, x]$ is always even where $f \in \operatorname{Aut}\left(D_{\infty}\right)$ and $x \in D_{\infty}$, it holds that $\left.\left[\operatorname{Aut}\left(D_{\infty}\right), D_{\infty}\right)\right] \cong \mathbb{Z}$ generated by ba. In fact, any power $(b a)^{k}$ for $k \in \mathbb{Z}$ can be written as a single commutator $[s, w]$, where $w$ is one of the two words of length $|k|$. Thus, $\mathrm{cl}_{\text {Aut }}$ is equal to one for any non-trivial element in $\left.\left[\operatorname{Aut}\left(D_{\infty}\right), D_{\infty}\right)\right]$ and $\operatorname{scl}_{\text {Aut }}$ vanishes.

Example 7.4 Consider $G=\operatorname{PSL}(2, \mathbb{Z})=\mathbb{Z} / 3 * \mathbb{Z} / 2$. Then $\operatorname{Aut}(G)$ is generated by the set $C$ consisting of the non-trivial factor automorphism of $\mathbb{Z} / 3$ and conjugations by letters of $\mathbb{Z} / 3$ and $\mathbb{Z} / 2$, since both free factors are abelian groups. Consequently, $[\operatorname{Aut}(G), G]$ is normally generated by commutators of the form $[c, g]=c g c^{-1} g^{-1}=c(g) g^{-1}$ for $c \in C$ and $g \in G$. In all expressions $[c, g]$ the letter $b$ representing the non-trivial element of the factor $\mathbb{Z} / 2$ arises an even number of times. Therefore, $b \notin[\operatorname{Aut}(G), G]$ and the latter is not the full group $G$. 
Lemma 7.5 Let $G=A * B$ be a free product of freely indecomposable groups where at least one of the factors is infinite cyclic. Then $[\operatorname{Aut}(G), G]$ has index at most two in $G$. Therefore, any unbounded quasimorphism on $G$ is unbounded when restricted to $[\operatorname{Aut}(G), G]$.

Proof If an unbounded quasimorphism $q$ is bounded on a finite index subgroup $H \leq G$, its homogenisation $\bar{q}$ vanishes on $H$. Then $\bar{q}$ descends to a map of sets on the finite set $G / H$ implying that the image of $\bar{q}$ is bounded and so was the image of $q$ to begin with. Therefore, any quasimorphism $q$ with unbounded image cannot be bounded on $H$. So, it remains to show that the index of $[\operatorname{Aut}(G), G]$ in $G$ is at most two to prove the lemma.

First, consider the case where $A$ and $B$ are both infinite cyclic and so $G$ can be identified with $F_{2}$, the free group of rank 2 . Let $x$ and $y$ be standard generators. Consider the automorphism $\varphi$ of $F_{2}$ defined by $\varphi(x)=y x$ and $\varphi(y)=y$. Then $[\varphi, x]=\varphi(x) \cdot x^{-1}=y$. So $\langle y\rangle \leq\left[\operatorname{Aut}\left(F_{2}\right), F_{2}\right]$. By symmetry of the generating set it holds that $\langle x\rangle \leq\left[\operatorname{Aut}\left(F_{2}\right), F_{2}\right]$ as well and it follows that $\left[\operatorname{Aut}\left(F_{2}\right), F_{2}\right]=F_{2}$.

Second, consider the case where only one of the factors is infinite cyclic. Without loss of generality assume $A=\mathbb{Z}$. Let $x$ denote a generator of $A$. For any $b \in B$ we can define the transvection $\varphi_{b}$ on $x$ by $\varphi_{b}(x)=b x$ and by $\varphi_{b}\left(b^{\prime}\right)=b^{\prime}$ for all $b^{\prime} \in B$. Then $\varphi_{b}$ is an automorphism and satisfies $\left[\varphi_{b}, x\right]=\varphi_{b}(x) \cdot x^{-1}=b$ for all $b \in B$. Thus, $B \leq[\operatorname{Aut}(G), G]$. Moreover, denoting the non-trivial factor automorphism of $A=\mathbb{Z}$ by $f$ we compute $[f, x]=$ $f(x) \cdot x^{-1}=x^{-2}$ and deduce that $2 \mathbb{Z} \leq[\operatorname{Aut}(G), G]$. Therefore, $2 \mathbb{Z} * B \leq[\operatorname{Aut}(G), G]$. Since [Aut $(G), G]$ is a normal subgroup, it holds that $N \leq[\operatorname{Aut}(G), G]$ where $N$ is the normal closure of $2 \mathbb{Z} * B$. However, $G / N \leq \mathbb{Z} / 2$ and so [Aut $(G), G$ ] has at most index 2 in $G$.

Proof of Theorem 2 If one of the factors is infinite cyclic, Theorem 1 implies the existence of an unbounded $\operatorname{Aut}(\mathrm{G})$-invariant homogeneous quasimorphism, which is unbounded on $[\operatorname{Aut}(G), G]$ according to Lemma 7.5. The statement then follows from Lemma 7.2.

Assume from now on that neither $A$ nor $B$ is infinite cyclic. We will prove the theorem by explicitly constructing an element in $[\operatorname{Aut}(G), G]$ together with homogeneous Aut-invariant quasimorphism which is non-trivial on that element.

The only non-trivial group with no non-trivial automorphisms is $\mathbb{Z} / 2$. Thus, one of the factors has to have a non-trivial automorphism since $A * B$ is not the infinite dihedral group. Without loss of generality we assume that $|\operatorname{Aut}(A)| \geq 2$. Let $a_{1} \in A$ such that $f\left(a_{1}\right)=a_{2} \neq a_{1}$ for some $f \in \operatorname{Aut}(A)$. Then we compute that $\left[f, a_{1}\right]=a_{2} a_{1}^{-1}$ is a non-trivial element in $[\operatorname{Aut}(G), G]$. Since $[\operatorname{Aut}(G), G]$ is normal, it holds for any $h \in B$ that $h a_{2} a_{1}^{-1} h^{-1} \in[\operatorname{Aut}(G), G]$. Thus, $\left(a_{2} a_{1}^{-1} h a_{2} a_{1}^{-1} h^{-1}\right)^{k} \in[\operatorname{Aut}(G), G]$ for any $k \in \mathbb{N}$ and $h \in B$.

First, assume that $A$ and $B$ are not isomorphic. Since $|\operatorname{Aut}(A)| \geq 2$, it holds that $|A| \geq 3$ and we can choose a non-trivial $a \neq a_{2} a_{1}^{-1}$ and fix some non-trivial $h \in B$. We define for $n_{1}, \ldots, n_{\ell} \in \mathbb{N}$ the word

$$
w=\prod_{i=1}^{\ell}\left([a, h]\left(a_{2} a_{1}^{-1} h a_{2} a_{1}^{-1} h^{-1}\right)^{n_{i}}\right) \in[\operatorname{Aut}(G), G] .
$$

We calculate

$$
\mathrm{A}-\operatorname{code}(w)= \begin{cases}\left(1,1,2 n_{1}, 1,1,2 n_{2}, \ldots, 1,1,2 n_{\ell}\right) & \text { if } a^{-1} \neq a_{2} a_{1}^{-1} \\ \left(1,2 n_{1}+1,1,2 n_{2}+1, \ldots, 1,2 n_{\ell}+1\right) & \text { if } a^{-1}=a_{2} a_{1}^{-1}\end{cases}
$$

Set $z=\mathrm{A}$-code $(w)$. For all $n \in \mathbb{N}$ it holds that $\mathrm{A}$-code $\left(w^{n}\right)=(z, \ldots, z)$. Choose $\ell \geq 3$ together with large and distinct $n_{1}, \ldots, n_{\ell} \in \mathbb{N}$, which implies that $z$ is generic. It follows from 
Proposition 4.11 that $\bar{f}_{z}^{A}$ is an Aut-invariant quasimorphism. By construction $f_{z}^{A}$ satisfies $f_{z}^{A}\left(w^{n}\right)=n$ for all $n \in \mathbb{N}$ and so $\bar{f}_{z}^{A}(w)>0$ for our choice of $w \in[\operatorname{Aut}(G), G]$. The statement then follows from Lemma 7.2.

Second, assume $A \cong B$. Let $s$ denote a swap automorphism. Set $b_{i}=s\left(a_{i}\right)$ for $i \in\{1,2\}$. Then the element $\left[s f s^{-1}, b_{1}\right]=b_{2} b_{1}^{-1} \in[\operatorname{Aut}(G), G]$ is non-trivial and belongs to the factor $B$. Set $b=s(a)$. Then $a^{-1}=a_{2} a_{1}^{-1}$ is equivalent to $b^{-1}=b_{2} b_{1}^{-1}$. For $n_{1}, \ldots, n_{\ell} \in \mathbb{N}$ we define the word

$$
w=\prod_{i=1}^{\ell}\left([a, b]\left(a_{2} a_{1}^{-1} b_{2} b_{1}^{-1}\right)^{n_{i}}\right) \in[\operatorname{Aut}(G), G] .
$$

Observe, that $\mathrm{A}-\operatorname{code}(w)=\mathrm{B}-\operatorname{code}(w)$. As in the previous case,

$$
\mathrm{A}-\operatorname{code}(w)= \begin{cases}\left(1,1, n_{1}, 1,1, n_{2}, \ldots, 1,1, n_{\ell}\right) & \text { if } a^{-1} \neq a_{2} a_{1}^{-1}, \\ \left(1, n_{1}+1,1, n_{2}+1, \ldots, 1, n_{\ell}+1\right) & \text { if } a^{-1}=a_{2} a_{1}^{-1} .\end{cases}
$$

Set $z=\mathrm{A}-\operatorname{code}(w)$ as before and choose $\ell \geq 3$ and $n_{1}, \ldots, n_{\ell} \in \mathbb{N}$ large enough and distinct, so that $z$ is generic. Again, we calculate $f_{z}^{A}\left(w^{n}\right)=n=f_{z}^{B}\left(w^{n}\right)$, which implies that $\left(\bar{f}_{z}^{A}+\bar{f}_{z}^{B}\right)(w)>0$. Proposition 4.11 implies that $\bar{f}_{z}^{A}+\bar{f}_{z}^{B}$ is an homogeneous Autinvariant quasimorphism and so applying Lemma 7.2 concludes the proof.

We will now give a few more examples of free products $G$ where $[\operatorname{Aut}(G), G]=G$ and therefore the notions of Aut-invariant (stable) commutator length are defined on all elements of $G$.

Example 7.6 For a product $G=A * B$ of two freely indecomposable perfect groups $A$ and $B$ it holds that $[\operatorname{Aut}(G), G]=G$. Indeed, since $A$ perfect, it holds that $A=$ $[A, A] \leq[\operatorname{Aut}(G), G]$. Similarly, $B \leq[\operatorname{Aut}(G), G]$ and $A$ and $B$ generate $G$ it follows that $[\operatorname{Aut}(G), G]=G$.

Example $7.7 G=\mathbb{Z} / p * \mathbb{Z} / q$ satisfies $[\operatorname{Aut}(G), G]=G$ for $p, q \geq 3$ prime. Let $m: \mathbb{Z} / p \rightarrow \mathbb{Z} / p$ be multiplication by 2 , which is a factor automorphism. Consider the standard generator $1_{p} \in \mathbb{Z} / p$. It holds that $\left[m, 1_{p}\right]=m\left(1_{p}\right)-1_{p}=1_{p}$. Thus, $\mathbb{Z} / p \leq[\operatorname{Aut}(G), G]$. Similarly, $\mathbb{Z} / q \leq[\operatorname{Aut}(G), G]$ and so $[\operatorname{Aut}(G), G]=G$.

Example 7.8 Let $k, \ell \geq 2$. Then $G=A^{k} * B^{\ell}$ satisfies [Aut $\left.(G), G\right]=G$ for all non-trivial abelian groups $A$ and $B$. For simplicity of notation consider the case $k=2$. Let $\phi$ be the factor automorphism defined by $\phi(a, 0)=(a, a)$ and $\phi(0, a)=a$ for all $a \in A$. Then $[\phi,(a, 0)]=\phi(a, 0)-(a, 0)=(0, a)$ for all $a \in A$. Thus, $A \times 0 \leq[\operatorname{Aut}(G), G]$. Analogously, $0 \times A \leq[\operatorname{Aut}(G), G]$. Since these factors generate $A^{2}$ it follows that $A^{2} \leq[\operatorname{Aut}(G), G]$. Similarly, $B^{\ell} \leq[\operatorname{Aut}(G), G]$ and so $[\operatorname{Aut}(G), G]=G$.

Example 7.9 In all of the above examples for $G=A * B$ the equality $[\operatorname{Aut}(G), G]=G$ is always derived by showing that both factors $A$ and $B$ form subgroups of $[\operatorname{Aut}(G), G]$. Thus, any combination of free factors appearing in the three examples above still satisfies this equality, for example $A=\mathbb{Z} / p$ for $p \geq 3$ prime and $B$ any freely indecomposable perfect group.

Acknowledgements I like to thank Jarek Kędra and Benjamin Martin for their continued support and all their helpful comments. This work was partly funded by the Leverhulme Trust Research Project Grant RPG-2017159. 
Open Access This article is licensed under a Creative Commons Attribution 4.0 International License, which permits use, sharing, adaptation, distribution and reproduction in any medium or format, as long as you give appropriate credit to the original author(s) and the source, provide a link to the Creative Commons licence, and indicate if changes were made. The images or other third party material in this article are included in the article's Creative Commons licence, unless indicated otherwise in a credit line to the material. If material is not included in the article's Creative Commons licence and your intended use is not permitted by statutory regulation or exceeds the permitted use, you will need to obtain permission directly from the copyright holder. To view a copy of this licence, visit http://creativecommons.org/licenses/by/4.0/.

\section{References}

1. J. Barge, E. Ghys, Cocycles d'Euler et de Maslov, Math. Ann. 294, 1992, 235-265.

2. C. Bavard. Longueur stable des commutateurs, Enseign. Math. (2) 37(1-2), 1991, 109-150.

3. M. Brandenbursky, M. Marcinkowski, Aut-invariant norms and Aut-invariant quasimorphisms on free and surface group, Commentarii Mathematici Helvetici, vol.94, No. 4, 2019, 661-687.

4. Robert Brooks, Some remarks on bounded cohomology, Riemann surfaces and related topics: Proceedings of the 1978 Stony Brook Conference (State Univ. New York, Stony Brook, N.Y., 1978) (Princeton, N.J.), Ann. of Math. Stud., vol. 97, Princeton Univ. Press, 1981, pp.53-63.

5. D. Calegari, K. Fujiwara, Stable commutator length in word-hyperbolic groups, Groups, Geometry and Dynamics 4 (no. 1), 2010, 59-90.

6. D. Calegari, scl. Mathematical Society of Japan Memoirs: Volume 20, 2009.

7. M. Entov, L. Polterovich, Calabi quasimorphism and quantum homology, International Mathematics Research Notices, 2003.

8. N.D. Gilbert, Presentations of the Automorphism Group of a Free Product, Proceedings of the London Mathematical Society, vol. 3-54, Issue 1, 1987, 115-140.

9. J.M. Gambaudo, E. Ghys Commutators and diffeomorphisms of surfaces, Ergodic Theory Dynam. Systems 24, no. 5, 2004, 1591-1617.

10. D.I. Fouxe-Rabinovitch, Über die Automorphismengruppen der freien Produkte I, Rec. Math. [Mat. Sbornik] vol. 8, 1940, 265-276.

11. D.I. Fouxe-Rabinovitch, Über die Automorphismengruppen der freien Produkte II, Rec. Math. [Mat. Sbornik] vol. 9, 1940, 183-220.

12. M. Kawasaki, M. Kimura $\hat{G}$-invariant quasimorphisms and symplectic geometry of surfaces, arxiv: 1911.10855, to appear in Israel Journal of Math.

13. M. Marcinkowski, Aut-Invariant Word Norm on Right-Angled Artin and Right-Angled Coxeter Groups Michigan Math. Journal, Volume 69, Issue 2 (2020), 285-295.

Publisher's Note Springer Nature remains neutral with regard to jurisdictional claims in published maps and institutional affiliations. 